\title{
Non-intervention observation: Dynamic evolution laws of inflammatory response in necrotizing enterocolitis
}

\author{
WEI WANG ${ }^{1}$, LI XUE $^{1}$, TONGSHENG MA ${ }^{2},{\text { YUAN } \mathrm{LI}^{3} \text { and ZHENGUANG LI }}^{1}$ \\ ${ }^{1}$ Neonatal Department of Internal Medicine, and Departments of ${ }^{2}$ Neonatal Surgery and ${ }^{3}$ Urology, \\ Xuzhou Children's Hospital, Xuzhou, Jiangsu 221002, P.R. China
}

Received February 2, 2016; Accepted July 25, 2016

DOI: $10.3892 / \mathrm{etm} .2016 .3540$

\begin{abstract}
The pathogenesis of necrotizing enterocolitis (NEC) is not well understood but immunological factors are thought to be key determinants of the disease appearance and its prognosis. During the course of the present study, different groups of newborn infants were observed and tested, to obtain an accurate image of values of pro- and anti-inflammatory cytokines at the onset, development and progression of neonatal NEC and to compare the values to those obtained during normal healthy development. All the infants in the study received standard medical treatment as appropriate. Initially, all the low birth weight premature infants born between June, 2014 and June, 2015 were tested on days 1, 3, 7, 10, 14, and 21 after birth, to obtain serum values of platelet activating factor (PAF), interleukin-1 (IL-1), tumor necrosis factor- $\alpha$ (TNF- $\alpha$ ) and IL-10. In total, 150 low birth weight premature infants were included, and the incidence of NEC was $6.67 \%$ (10/150). For the comparison studies, 10 premature NEC infants of low birth weight, 15 premature normal infants, and 15 full-term normal infants born during the same period were enrolled in the study. The serum values of PAF, IL-1, TNF- $\alpha$ and IL-10 for these infants were detected on the same days after birth. PAF, IL-1 and TNF- $\alpha$ levels began to increase on days 1-3 after birth in premature infants, reached a peak on days 7-10, and declined to normal levels on days 14-21. Comparison differences in premature and full-term infants were statistically significant $(\mathrm{P}<0.01)$. Interleukin-10 began to increase on days 7-10 after birth in premature infants and reached a peak on days 14-21. Comparisons among premature and fullterm infants at the given time points showed the differences were also statistically significant $(\mathrm{P}<0.01)$. The differences in values of the above inflammatory cytokines in the infants
\end{abstract}

Correspondence to: Dr Zhenguang Li, Neonatal Department of Internal Medicine, Xuzhou Chlidren's Hospital, 18 Sudibei Road, Xuzhou, Jiangsu 221002, P.R. China

E-mail: zhenguangli29@163.com

Key words: inflammatory response, neonatal necrotizing enterocolitis, platelet activating factor, tumor necrosis factor- $\alpha$, interleukin-1, interleukin-10 that died and the values in the survivors were not statistically significant $(\mathrm{P}>0.05)$. In conclusion, pro-inflammatory factors PAF, IL-1, TNF- $\alpha$ and anti-inflammatory factor IL-10 may be important in the pathogenesis of NEC, and monitoring their levels in blood can be useful in the prediction of the occurrence of disease. Nevertheless, these levels are not useful as prognostic markers.

\section{Introduction}

Neonatal necrotizing enterocolitis (NEC) is a common disease of newborn infants. Among premature infants of low birth weight, the incidence of NEC is particularly high, the morbidity rate is $1.6-7.3 \%$ and mortality can be as high as $50-80 \%$ (1). Although animal experiments and clinical studies have been conducted to investigate NEC, its exact pathogenesis remains to be determined, and a more sensitive diagnostic method and effective treatment approach have yet to be identified. Previous results repeatedly stress that an inflammatory cascade reaction is, not only a starting factor of NEC, but also plays an important role in the evolution and prognosis of NEC (2). The roles of platelet-activating factor (PAF), interleukins (IL-1, IL-10) and tumor necrosis factor- $\alpha$ (TNF- $\alpha$ ) have been confirmed in NEC animal models and their corresponding antagonists can be useful to reduce the disease damage and improve clinical effects (3). Furthermore, associated signal transduction pathways, such as signal peptide RhoA and Toll-like receptors, are involved in the occurrence of NEC (4).

However, since scientific and comprehensive data at present remain scarce, these inflammatory factors are rarely used in clinical diagnosis, treatment and prognosis evaluation. Most clinical studies focus on the detection of inflammation indexes in infants that have been diagnosed with NEC, and there are few detection time points (5). In the present study, through rigorous experimental design and careful observation of the evolution of the inflammatory process prior to and at the onset of NEC, PAF, IL-1, TNF- $\alpha$ and IL-10 were found to be important in the pathogenesis of NEC, albeit their levels were not useful as prognostic markers.

\section{Patients and methods}

Patients. In total, 150 premature infants of low birth weight born at the Xuzhou Children's Hospital between 
June, 2014 and June, 2015 were included in the study. All 150 infants were caesarean delivery babies and met the inclusion criteria: i) healthy mothers (no hypertension, diabetes, heart disease or other infectious diseases, no history of abortion, premature birth, or stillbirth); ii) no innate or acquired deformities (no injuries or conscious disturbances); and iii) one minute Apgar scores $\geq 7$. The exclusion criteria were defined and listed as: i) withdrawing from the study, treatment suspension or transfer to another hospital; and ii) death within $24 \mathrm{~h}$ after birth, severe asphyxia, low body temperature or other complications.

Approval for the study was obtained from the Ethics Committee of the Xuzhou Children's Hospital. Written informed consent was obtained from the legal guardians.

During the trial, 10 infants were diagnosed with NEC (NEC group). In addition, 15 premature normal infants (caesarean delivery babies) were included in the premature normal group and 15 full-term normal infants were enrolled into the full-term normal group. The NEC group comprised 6 male and 4 female infants, the gestational age at birth

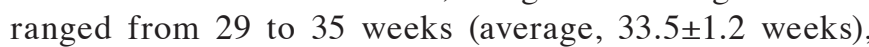
and the infant weight ranged from 1.3 to $1.6 \mathrm{~kg}$ (average, $1.5 \pm 0.2 \mathrm{~kg}$ ). The premature normal group included 8 male and 7 female infants, the gestational age at birth ranged from 28 to 36 weeks (average, $34.2 \pm 1.3$ weeks), and the infant weights were $1.3-1.6 \mathrm{~kg}$ (average, $1.5 \pm 0.4 \mathrm{~kg}$ ). No significant differences in gender, age or body weight were observed between the two groups $(\mathrm{P}>0.05)$. The full-term normal group included 9 male and 6 female infants, and their weights ranged from 2.8 to $3.3 \mathrm{~kg}$ (average, $3.0 \pm 0.3 \mathrm{~kg}$ ). Of the 15 infants in this group, 12 were normal deliveries and 3 were caesarean delivery babies.

Methods. The serum value for PAF, IL-1, TNF- $\alpha$ and IL-10 for the infants included in the study, were taken on days $1,3,7,10$, 14, and 21 after birth. Premature and NEC infants were treated according to the standard medical regimes needed for their care.

The specialized equipment used in the study included a microplate reader (Model 352; Finland Labsystems Multiskan, Helsinki, Finland), a microplate washer (Model AC8; Thermo Labsystems, Helsinki, Finland), and a TG16W high speed micro-centrifuge. The reagent kits for ELISA detection of PAF, IL-1, TNF- $\alpha$ and IL-10 from blood samples were purchased from BD Biosciences Co. (San Diego, CA, USA). The experiments were carried out strictly in accordance with the protocols of the reagent kits.

Venous blood samples $(5 \mathrm{ml})$ were submitted for inspection, centrifugation at 2,000 x g for $15 \mathrm{~min}$, quiescence, and supernatant collection at the hospital's laboratory. Interleukin-1, TNF- $\alpha$ and IL-10 were detected by ELISA. Specifically, purified antibodies were used to coat microporous plates, and thus turn them into solid phase carrier plates. Antigen-containing serum samples were incubated with enzyme-linked affinity detection antibodies (IL-1: rabbit polyclonal antibody with a dilution of 1:500, catalog no.: ab2105; TNF- $\alpha$ : rabbit polyclonal antibody with a dilution of 1:500, catalog no.: ab6671; and IL-10: rabbit polyclonal antibody with a dilution of 1:500; catalog no.: ab100549). The enzyme-linked antigen complexes were transferred to the carrier-coated microporous plates to compete for the solid phase antibody present on the micropores. Once sufficient time was allowed for the labeled antigen-antibody complexes to form, the enzymatic substrates were added and a colorimetric reaction occurred. The substrate became blue under the effect of enzyme and then turned yellow under the effect of acid. Absorbance values were read on a micoplate reader, a standard curve was drawn, and the antigen concentrations of the samples were calculated.

A bioanalysis was conducted to detect PAF. Based on the strong promotion of platelet aggregation and 5-hydroxytryptamine release by PAF, a platelet aggregometer calculated the content of PAF in samples according to a standard curve of the platelet aggregation ratio. The reference ranges used in the present study were: $16.9-100.0 \mu \mathrm{g} / 1$ for PAF, $0.13-0.25 \mu \mathrm{g} / 1$ for IL-1, 5-100 ng/l for TNF- $\alpha$, and 28-49.2 $\mu \mathrm{g} / 1$ for IL-10.

Statistical analysis. Statistical software package SPSS 20.0 (Chicago, IL, USA) was used to process the data. The measurement data were presented as mean $\pm \mathrm{SD}$, and repeated measurement data were compared among the groups. Countable data were presented as a case or percentage, and the $\chi^{2}$ test was applied in comparisons in groups. $\mathrm{P}<0.05$ was considered statistically significant.

\section{Results}

Comparison of the dynamic evolution of inflammatory factors among the groups. The sets of values obtained for each group at different given days were compared in the different groups (Tables I-IV). Levels of PAF, IL-1 and TNF- $\alpha$ in the premature infants began to increase on days 1-3 after birth, reached a peak on days 7-10, and decreased on days 14-21 to close to normal levels. The differences observed between the premature and full-term infant groups were statistically significant $(\mathrm{P}<0.01)$ (Tables I-III). Interleukin-10 began to increase in the premature infant groups on days 7-10 after birth and reached a peak on days 14-21. The differences observed between the full-term and premature infant groups were statistically significant $(\mathrm{P}<0.01)$ (Table IV).

No significant differences were observed in the values between the infants affected by NEC who died and those who survived. Of note, there was a statistically significant difference in the values of inflammatory markers between the premature infants affected with NEC and those spared $(\mathrm{P}>0.05)$. After the first 6 cases of NEC, the subsequent 4 cases were predictable based on those differences.

Association between inflammatory factors and prognosis. Four the 10 NEC infants died despite receiving appropriate medical attention in hosptial, while the remaining six infants survived. The results suggest it may be possible to predict the development of NEC based on the abovementioned inflammatory factors detected in the sera of premature infants. However, the same factors cannot be reliably employed in the prognosis of the disease.

\section{Discussion}

At present, the pathogenesis and specific mechanisms of NEC remain to be elucidated. However, it is considered to be a result of various factors acting at the same time. Newborns with 
Table I. Comparison of the dynamic evolution of PAF among groups $(\mu \mathrm{g} / \mathrm{l})$.

\begin{tabular}{|c|c|c|c|c|c|c|}
\hline Group/day & 1 & 3 & 7 & 10 & 14 & 21 \\
\hline NEC & $125.2 \pm 35.7$ & $135.7 \pm 36.5$ & $366.8 \pm 54.7$ & $384.5 \pm 59.8$ & $235.6 \pm 42.3$ & $138.5 \pm 41.2$ \\
\hline Premature normal & $82.3 \pm 26.9$ & $92.8 \pm 32.4$ & $95.6 \pm 33.6$ & $115.4 \pm 37.8$ & $84.5 \pm 29.4$ & $87.8 \pm 30.2$ \\
\hline Full-term normal & $46.2 \pm 16.4$ & $48.5 \pm 17.2$ & $52.3 \pm 15.3$ & $53.4 \pm 14.6$ & $46.8 \pm 13.3$ & $44.5 \pm 15.5$ \\
\hline Dead & $124.2 \pm 32.3$ & $136.8 \pm 35.8$ & $382.4 \pm 52.6$ & $381.2 \pm 57.8$ & $246.5 \pm 41.3$ & $142.5 \pm 42.3$ \\
\hline Survivor & $127.3 \pm 31.5$ & $133.5 \pm 34.2$ & $359.7 \pm 53.2$ & $394.8 \pm 58.2$ & $229.8 \pm 41.6$ & $135.6 \pm 42.5$ \\
\hline
\end{tabular}

PAF, platelet-activating factor; NEC, necrotizing enterocolitis.

Table II. Comparison of the dynamic evolution of IL-1 among groups $(\mu \mathrm{g} / \mathrm{l})$.

\begin{tabular}{|c|c|c|c|c|c|c|}
\hline Group/day & 1 & 3 & 7 & 10 & 14 & 21 \\
\hline NEC & $0.26 \pm 0.03$ & $0.28 \pm 0.04$ & $0.85 \pm 0.05$ & $0.89 \pm 0.06$ & $0.43 \pm 0.03$ & $0.31 \pm 0.02$ \\
\hline Premature normal & $0.22 \pm 0.03$ & $0.23 \pm 0.02$ & $0.26 \pm 0.04$ & $0.27 \pm 0.03$ & $0.24 \pm 0.02$ & $0.21 \pm 0.01$ \\
\hline Full-term normal & $0.17 \pm 0.02$ & $0.18 \pm 0.03$ & $0.19 \pm 0.03$ & $0.20 \pm 0.04$ & $0.16 \pm 0.02$ & $0.15 \pm 0.02$ \\
\hline Dead & $0.25 \pm 0.02$ & $0.28 \pm 0.03$ & $0.83 \pm 0.05$ & $0.93 \pm 0.06$ & $0.42 \pm 0.08$ & $0.33 \pm 0.04$ \\
\hline Survivor & $0.26 \pm 0.01$ & $0.27 \pm 0.02$ & $0.92 \pm 0.06$ & $0.86 \pm 0.07$ & $0.46 \pm 0.05$ & $0.30 \pm 0.02$ \\
\hline
\end{tabular}

IL-1, interleukin-1; NEC, necrotizing enterocolitis.

Table III. Comparison of the dynamic evolution of tumor necrosis factor- $\alpha$ among groups ( $\mu \mathrm{g} / \mathrm{l})$.

\begin{tabular}{|c|c|c|c|c|c|c|}
\hline Group/day & 1 & 3 & 7 & 10 & 14 & 21 \\
\hline NEC & $94.5 \pm 32.4$ & $115.2 \pm 36.3$ & $175.6 \pm 40.2$ & $232.1 \pm 46.8$ & $146.8 \pm 26.7$ & $106.9 \pm 24.5$ \\
\hline Premature normal & $66.3 \pm 21.5$ & $82.5 \pm 25.6$ & $97.4 \pm 32.1$ & $120.5 \pm 37.8$ & $86.9 \pm 22.6$ & $74.7 \pm 23.4$ \\
\hline Full-term normal & $32.6 \pm 15.3$ & $33.7 \pm 15.4$ & $42.5 \pm 16.2$ & $43.6 \pm 16.5$ & $35.2 \pm 14.8$ & $30.1 \pm 15.2$ \\
\hline Dead & $92.2 \pm 31.4$ & $117.5 \pm 35.8$ & $180.3 \pm 36.8$ & $239.6 \pm 43.6$ & $142.5 \pm 23.8$ & $103.5 \pm 21.3$ \\
\hline Survivor & $96.3 \pm 31.6$ & $112.3 \pm 34.2$ & $172.4 \pm 38.4$ & $228.7 \pm 42.5$ & $150.3 \pm 23.5$ & $108.5 \pm 22.1$ \\
\hline
\end{tabular}

TNF- $\alpha$, tumor necrosis factor- $\alpha$; NEC, necrotizing enterocolitis.

Table IV. Comparison of the dynamic evolution of IL-10 among groups $(\mu \mathrm{g} / \mathrm{l})$.

\begin{tabular}{|c|c|c|c|c|c|c|}
\hline Group/day & 1 & 3 & 7 & 10 & 14 & 21 \\
\hline NEC & $48.5 \pm 10.2$ & $50.2 \pm 11.3$ & $62.5 \pm 12.2$ & $68.9 \pm 12.7$ & $94.2 \pm 14.5$ & $103.5 \pm 15.6$ \\
\hline Premature normal & $47.7 \pm 11.6$ & $48.2 \pm 12.4$ & $49.3 \pm 12.9$ & $50.2 \pm 13.2$ & $51.3 \pm 13.6$ & $52.4 \pm 13.5$ \\
\hline Full-term normal & $31.3 \pm 11.2$ & $32.6 \pm 11.3$ & $33.4 \pm 11.6$ & $34.5 \pm 11.4$ & $35.6 \pm 12.2$ & $36.6 \pm 12.5$ \\
\hline Dead & $50.2 \pm 9.6$ & $48.9 \pm 9.5$ & $64.3 \pm 10.2$ & $67.5 \pm 10.6$ & $96.6 \pm 11.4$ & $102.4 \pm 12.5$ \\
\hline Survivor & $47.2 \pm 9.7$ & $51.2 \pm 9.4$ & $60.3 \pm 10.3$ & $69.7 \pm 10.5$ & $93.3 \pm 11.3$ & $105.6 \pm 12.4$ \\
\hline
\end{tabular}

IL-1, interleukin-1; NEC, necrotizing enterocolitis.

asphyxia, hypoxia, hypotension, acidosis, and shock upon birth, are prone to defensive reflex blood redistribution, decreased mesenteric blood flow and intestinal mucosal ischemia and necrosis (6). Furthermore, the weakened intestinal mucosal barrier functions result in the propagation and translocation of a large number of intestinal pathogenic bacteria, and 
the release of a large number of toxic substances, such as endotoxin, IL-6, TNF-, and IL-4 (7). These inflammatory factors also promote the cascade reaction of intestinal mucosal inflammation, cause more extensive mucosal necrosis, enable more toxic substances to enter into the circulation of the blood, and lead to bacteremia, sepsis and shock (8).

It is possible that the first inflammatory factors secreted in NEC patients are TNF- $\alpha$ and PAF (9). When the homeostatic environment of the patient is disrupted, macrophages become activated and produce and release large amounts of TNF- $\alpha$, which, combined with specific receptors, produces various biological effects, leading to mitochondrial dysfunction, and ultimately mitochondrial apoptosis (10). After bacterial lipopolysaccharide and TNF- $\alpha$ injections into animal models, the animals develop intestinal necrosis. However, the degree of intestinal necrosis can be greatly alleviated by using pentoxifylline to inhibit TNF- $\alpha$ (11). PAF is an inflammatory cytokine that can lead to epithelial cell injury and apoptosis, and promote leukocyte and platelet aggregation, blood vessel contraction, and increased permeability (12). The addition of PAF-AH in infant formula can reduce the incidence of NEC. It is also known that premature infants lack endogenous PAF-AH. Thus, PAF is important in the pathogenesis of NEC (13). The results of the present study have shown that the levels of PAF and TNF- $\alpha$ in NEC infants began to increase on days 1-3 after birth, increasing by 50 and $45 \%$ of the initial value, respectively; the values reached a peak on days $7-10$, increasing by 280 and $80 \%$; and then declining on days 14-21 to almost normal levels. PAF and TNF levels in the NEC group were significantly higher than those in the normal preterm group, and those in the full-term normal group were the lowest of all the levels. There were no high values in the premature or full-term normal groups.

TNF- $\alpha$ and PAF probably induced the release of IL-1, and the high concentrations of IL- 1 and TNF- $\alpha$ jointly stimulated the body to release IL-6. These inflammatory factors interact with each other and form a feedback mechanism that can lead to the release of more inflammatory factors and the formation of a reaction cascade (14). Some scholars even believe that IL-1 can be used as a marker for reflecting cell factor cascade activation, revealing the association between disease severity and inflammatory response, and as a marker for sepsis and SIRS prognosis (15). At the same time, the expression levels of IL-11, and IL-10, as well as related receptors gradually increase, which may be useful to alleviate the excessive inflammatory response and NEC damages (16).

The results of the present study have shown the levels of IL-1 began to increase on days 1-3 after birth in premature infants, increasing by approximately $18 \%$; reached a peak on days $7-10$, increasing by approximately $230 \%$; and then declined on days 14-21 to close to normal levels. Interleukin-1 levels in the NEC group were significantly higher than those in the normal preterm group, and the full-term normal group had the lowest levels. Interleukin-10 began to increase on days 7-10 after birth, increasing by approximately 30\%; and reached a peak on days 14-21, increasing by approximately $90 \%$. Interleukin-10 levels in the NEC group were significantly higher than those in the normal preterm group, and the lowest values were found in the normal group.
In the present study, we monitored the fluctuations in the values of serum inflammatory factors within the first three weeks of life of infants and observed a clear difference between the values of full-term and premature infants. Of note, we found a statistically significant difference between the immature infants who went on to develop NEC and those who did not develop NEC. Thus, the present findings set a firm base showing that given an appropriate sample size, it is possible to obtain a reference range for the development of NEC in infants with high sensitivity and specificity. Such a reference index may provide a simple and powerful clinical tool for the early prediction of NEC.

Nevertheless, the present results also found that the prognosis of NEC cannot depend on the monitoring of any of the inflammatory factors tested, as it was found that none of the four indicators compared in the NEC survival and death group, showed statistical differences. The present study did not include a regression analysis of the prognostic factors. Thus, it was hypothesized that the prognosis may not only be associated with the level of inflammation, but also with the severity of the disease, the infants' basic state and the related treatment methods $(17,18)$. Therefore, the value of other inflammatory factors in the prediction of prognosis cannot yet be excluded.

In conclusion, the pro-inflammatory factors PAF, IL-1, TNF- $\alpha$ and the anti-inflammatory factor IL-10 may be involved in the pathogenesis of NEC, and monitoring their presence in the patient's blood can be used as a key to predict the occurrence of NEC. However, these factors may not prove useful in the prognosis of the disease.

\section{References}

1. Keshari RS, Jyoti A, Dubey M, Kothari N, Kohli M, Bogra J, Barthwal MK and Dikshit M: Cytokines induced neutrophil extracellular traps formation: implication for the inflammatory disease condition. PLoS One 7: e48111, 2012.

2. Nagy B, Timár G, Józwiak-Hagymásy J, Kovács G, Merész G, Vámossy I, Ágh T, László Á, Vokó Z and Kaló Z: The cost-effectiveness of ulipristal acetate tablets in treating patients with moderate to severe symptoms of uterine fibroids. Eur J Obstet Gynecol Reprod Biol 175: 75-81, 2014.

3. Tristan M, Orozco LJ, Steed A, Ramírez-Morera A and Stone P: Mifepristone for uterine fibroids. Cochrane Database Syst Rev 8: CD007687, 2012.

4. Afrazi A, Sodhi CP, Richardson W, Neal M, Good M, Siggers R and Hackam DJ: New insights into the pathogenesis and treatment of necrotizing enterocolitis: Toll-like receptors and beyond. Pediatr Res 69: 183-188, 2011.

5. Gregoric P, Sijacki A, Stankovic S, Radenkovic D, Ivancevic N, Karamarkovic A, Popovic N, Karadzic B, Stijak L, Stefanovic B, et al: SIRS score on admission and initial concentration of IL-6 as severe acute pancreatitis outcome predictors. Hepatogastroenterology 57: 349-353, 2010.

6. Garg PM and Shekhawat P: Transfusion-associated necrotizing enterocolitis: authors reply. Indian Pediatr 53: 537-538, 2016.

7. Yu L, Tian J, Zhao X, Cheng P, Chen X, Yu Y, Ding X, Zhu X and Xiao Z: Bowel perforation in premature infants with necrotizing enterocolitis: risk factors and outcomes. Gastroenterol Res Pract 2016: 6134187, 2016

8. Alexander VN, Northrup V and Bizzarro MJ: Antibiotic exposure in the newborn intensive care unit and the risk of necrotizing enterocolitis. J Pediatr 159: 392-397, 2011.

9. Yousef AA, Amr YM and Suliman GA: The diagnostic value of serum leptin monitoring and its correlation with tumor necrosis factor-alpha in critically ill patients: a prospective observational study. Crit Care 14: R33, 2010.

10. Tayman C, Aydemir S, Yakut I, Serkant U, Ciftci A, Arslan E and Koç O: TNF- $\alpha$ blockade efficiently reduced severe intestinal damage in necrotizing enterocolitis. J Invest Surg 18: 1-9, 2016. 
11. .Jia SH, Wei H, Yu JL, Wei XD, Zhang XP and Li JC: Protective effects of curcumin on neonatal rats with necrotizing enterocolitis. Zhongguo Dang Dai Er Ke Za Zhi 12: 132-136, 2010 (In Chinese).

12. Sankararaman S, Yanamandra K, Napper D, Caldito G and Dhanireddy R: The prevalence of platelet activating factor acetylhydrolase single nucleotide polymorphisms in relationship to necrotizing enterocolitis in Northwest Louisiana infants. Springerplus 2: 294, 2013.

13. Froeling V, Scheurig-Muenkler C, Hamm B and Kroencke TJ: Uterine artery embolization to treat uterine adenomyosis with or without uterine leiomyomata: results of symptom control and health-related quality of life 40 months after treatment. Cardiovasc Intervent Radiol 35: 523-529, 2012.

14. Dinarello CA: Interleukin-1 in the pathogenesis and treatment of inflammatory diseases. Blood 117: 3720-3732, 2011.

15. Sikora JP, Kuzański W and Andrzejewska E: Soluble cytokine receptors sTNFR I and sTNFR II, receptor antagonist IL-1ra, and anti-inflammatory cytokines IL-10 and IL-13 in the pathogenesis of systemic inflammatory response syndrome in the course of burns in children. Med Sci Monit 15: CR26-CR31, 2009.
16. Cöl R and Durgun Z: Effect of recombinant interleukin-10 on some haematological and biochemical parameters in a rat endotoxaemic model. Acta Vet Hung 59: 237-245, 2011.

17. Borthakur A, Bhattacharyya S, Alrefai WA, Tobacman JK, Ramaswamy K and Dudeja PK: Platelet-activating factor-induced $\mathrm{NF}-\kappa \mathrm{B}$ activation and IL-8 production in intestinal epithelial cells are Bcl10-dependent. Inflamm Bowel Dis 16: 593-603, 2010.

18. Maynard AA, Dvorak K, Khailova L, Dobrenen $\mathrm{H}$, Arganbright KM, Halpern MD, Kurundkar AR, Maheshwari A and Dvorak B: Epidermal growth factor reduces autoghagy in intestinal epithelium and in the rat model of necrotizing enterocolitis. Am J Physiol Gastrointest Liver Physiol 299: 614-622, 2010 . 